\title{
Evaluación interna y externa \\ de la Revista Enfermería Universitaria \\ de la ENEO-UNAM durante \\ el período 2004-2006.
}

\author{
Assessment of internal and external review of nursing \\ college eneo-unam for the period 2004-2006.
}

Dra. Lasty Balseiro Almario, * Mtra. María de los Ángeles Torres Lagunas

$y$ ** Mtra. Rosa A. Zárate Grajales. **

*Profesora de Carrera Titular "B" de la ENEO-UNAM.

**Profesoras de Carrera Asociadas "C" de la ENEO-UNAM

\section{Resumen}

Objetivo: La finalidad de la presente investigación fue evaluar de forma interna y externa la revista Enfermería Universitaria de la ENEO-UNAM en el periodo comprendido del 2004-2006, a fin de identificar la calidad científica y editorial de la revista. Metodología: La variables a identificar son las siguientes; de productividad, de estabilidad y obsolescencia, de calidad editorial, de calidad científica y de infraestructura. Tomando como base cada uno de estos indicadores se realizaron las preguntas de los cuestionarios. El tipo de investigación fue descriptivo, analítico, retrospectivo, longitudinal y de corte cuantitativo. El diseño fue no experimental. Los cuestionarios utilizados fueron de tres tipos: una guía de indicadores de los para los 9 ejemplares de la revista y dos cuestionarios para la evaluación externa, uno de ellos para las y los autores y el otro para las y los lectores. La aplicación de los cuestionarios fue para 50 autores y para 116 lectores. La investigación se realizó en el año 2007. Resultados: En la evaluación externa, el $90.5 \%$ de los autores fueron enfermeras en docencia e investigación y de los lectores, el 98\% son enfermeras en administración, docencia e investigación. En cuanto a la calidad científica del $70-81 \%$ de las y los lectores y autoras/es refirió que la revista es un medio de comunicación primordial para enfermería que aporta información relevante. En relación con la calidad editorial entre el 75-85\% de las y los lectores y autoras/es indicó que los artículos son evaluados por pares, arbitrados localmente de manera anónima. En lo referente al diseño editorial, entre el 50 y el 99\%, el diseño informativo es el adecuado pero requiere mejoras. La escala de grises y el tipo de letra es apropiada. Los espacios son suficientes. De la evaluación interna, se publicaron 9 ejemplares 80 artículos en siete secciones principalmente por profesorado y alumnado de la ENEO-UNAM y en una mínima proporción por autoras/ es de otras Universidades e Instituciones de salud del país y del extranjero. Se citaron 613 referencias: 210 de enfermería y 403 de otros profesionales de la salud, el número de citas promedio por artículo por ejemplar fue de 7 con un intervalo de 3.9 a 10.4. El idioma en que se cita fue predominantemente el español, el estilo de elaboración de citas es Vancouver. La revista a su corta edad es un vínculo de comunicación del pensamiento de enfermería en el país, fue publicada cuatrimestralmente y se constituyó en un recurso valioso para el desarrollo del conocimiento de la disciplina de Enfermería en México. Conclusiones: La evaluación realizada arrojó datos positivos. Sin embargo, la revista debe ser mejorada y fortalecida en todas su debilidades para lograr la calidad científica y editorial.

PALABRAS CLAVE: Evaluación, revistas, enfermería, bibliometría, visibilidad, obsolescencia, calidad científica, calidad editorial e infraestructura, Indicadores, autores y lectores. 


\section{Abstract}

Objective: The purpose of this research was to analyze the internal and external evaluation of the college of nursing journal ENEO-UNAM in the period of 2004-2006, to assess the scientific and publishing the magazine. Methodology: The identify the variables are as follow; productivity, stability and obsolescence of editorial quality and infrastructure. Each of these dimensions was measured with their indicators. Based on each of these indicators were the questions related to the questionnaires. The type of research was descriptive, analytical, retrospective, Iongitudinal and cutting quantity. The design was not experimental. The questionnaires were used in three types: a guide to indicators of the 9 copies of the magazine and two questionnaires for the external evaluation, one of them and to the authors and to the readers. The implementation of the questionnaires was for 50 to authors and 116 to readers. The research was conducted in 2007. Results: In the Internal evaluation, the $90.5 \%$ of the perpetrators was nurses in teaching and research. Of the readers $98 \%$ in nursing administration, teaching and research. In terms of scientific quality $70-81 \%$ of the readers and authors are said that the magazine is a primary means of communication for nursing that brings relevant information. In connection with the editorial quality between $75-85 \%$ of the readers and authors indicated that the items are evaluated by peers, locally arbitrated on an anonymous basis. Regarding the editorial design, between 50 and 99\%, design information is adequate but needs improvement. The gray scale and the font are appropriate. The spaces are sufficient. Of internal evaluation, 9 copies were published 80 articles in seven sections, mostly by faculty and students from the UNAM-ENEO and a minimum proportion by the authors from other universities and institutions of health of the country and abroad. 613 references were cited: 210 nurses and 403 other health professionals, the average number of citations per article was 7 per copy with a range of 3.9 to 10.4 . The language in which it cited was predominantly Spanish, the style of developing are Vancouver. The magazine to his young age he was thinking of the communication link of nurses in the country, was published quarterly. Was founded in those years into a valuable resource for the development of knowledge of the discipline of Nursing in Mexico. Conclusions: The assessment carried out showed positive data. However, the magazine should be improved and strengthened in all its weaknesses to achieve scientific and editorial.

KEY WORDS: Assessment, magazines, nursing bibliometría, visibility, obsolescence, scientific quality, editorial quality and infrastructure, indicators, authors and readers.

\section{INTRODUCCIÓN}

La presente investigación tiene por objeto analizar la evaluación interna y externa de la Revista Enfermería Universitaria de la ENEO en el período 2004-2006 con el fin de determinar la calidad editorial y científica de la misma.

Problema: El problema se enunció de la siguiente forma: ¿Cuáles son los resultados de la evaluación interna y externa de la revista Enfermería Universitaria de la ENEO-UNAM, durante los años 2004, 2005 y 2006?. Las respuestas obtenidas permitirán establecer de la calidad editorial y científica de la revista.

Objetivos: El objetivo general fue realizar un diagnóstico de la producción científica de la Revista Enfermería-Universitaria de la ENEO-UNAM a partir de los resultados de la evaluación interna y externa durante los años 2004, 2005 y 2006. Cómo objetivos específicos se propusieron analizar la productividad científica de la revista Enfermería Universitaria en ese mismo período de tiempo; otros objetivos fueron: identificar los indicadores bibliométricos de la revista, la estabilidad, la calidad editorial y la calidad científica en el mismo lapso de tiempo.

\section{MARCO TEORICO}

Para Manuel Amezcua, la construcción de la ciencia implica un proceso complejo, cuyo resultado final es una publicación. Así, la ciencia se da a conocer a partir de la publicación científica, ya que este es el medio para difundir los conocimientos, contrastar los hallazgos encontrados y utilizar estos hallazgos en la construcción teórica o práctica de la ciencia'. La ciencia entonces, es un producto que se publica y se remite al estudio de una actividad llamada científica que refleja el pensamiento de esta comunidad, que pone al servicio de todos los hallazgos encontrados. Por ello, en una comunidad internacional que está globalizada el conocimiento científico está al alcance de todos. Esto hace que el producto científico sea excluído de algunas comunidades científicas y de algunas áreas de la ciencia del circuito general del conocimiento. Este es un hecho muy común en el área de Enfermería ya que se escribe en español pero el producto científico no llega a un conjunto de comunidades científicas, académicas y profesionales nacionales e internacionales de varios continentes, lo que conlleva a obtener un producto científico muy variable, ya que puede ser 
grande para unas comunidades y pequeño para otras. Es así de esta forma como nace, la gran ciencia y la ciencia poco trascendente.

De la relación de la enfermería con la ciencia se destacan dos apartados: el lugar que ocupa la enfermería dentro de la ciencia y el conocimiento de la enfermería en español en el contexto internacional versus el conocimiento de la enfermería internacional. Así, el producto generado por la actividad científica de la enfermería española por ejemplo, tiene el comportamiento de una ciencia aplicada y práctica, pero en la UNESCO se le clasifica como "Ciencia Médica" y experimental. No obstante, la mayoría de las revistas de enfermería incluidas en los índices de citas no se encuentran en el Sciece Citation Index (SCl)2. Esta contradicción se explica por la diversidad de las fuentes, paradigmas y métodos usados en la investigación. Así, el hablar del conocimiento de la enfermería internacional no es hablar de la enfermería en español; por lo que se publica en español es equivalente a no internacional.

\section{El análisis documental como método de evaluación de la ciencia de enfermería.}

El análisis documental para Manuel Amezcua, es el marco teórico para realizar la evaluación del conocimiento y dentro de ello, el estudio de los indicadores de la actividad científica basados en variables bibliométricas y bibliográficas. Estas variables, dentro de una perspectiva crítica expresan la prudencia interpretativa de los hallazgos de los estudios documentales. De esta experiencia se destacan dos consideraciones: la adecuada contextualización del campo de la ciencia estudiada con la necesidad de un enfoque ecléctico y la rigurosidad de sus indicadores de actividad para ese contexto ${ }^{3}$. La primera de las recomendaciones hace referencia al campo de la ciencia objeto de estudio y en segundo lugar, hay que reseñar la dimensión cronológica de la investigación en enfermería de esta área logística de la que destaca su alta complejidad dada la diversidad de hechos geopolíticos que rodean a las distintas comunidades científicas nacionales.

\section{Las revistas que publican investigaciones en Enfermería.}

Para Raquel Benavides Torres la práctica basada en la investigación es vista como fundamental para el desarrollo profesional y por lo tanto, para la práctica autónoma de enfermería. Es de suma importancia que las enfermeras puedan evidenciar y describir su contribución profesional a la salud de los individuos, grupos, comunidades y población en general desarrollando investigaciones centradas principalmente en el cuidado de enfermería, para el establecimiento de un cuerpo de conocimientos propios. ${ }^{4}$ Así, la investigación en enfermería es todavía una actividad desdibujada, en donde pocos se dedican a ella, pero también, pocos son los que tienen un grado académico avanzado que les permita explorar problemas para generar teorías o modelos de práctica innovadoras. La mayor parte de la investigación es realizada por académicos universitarios y el volumen de la publicación es escaso, lo cual limita el conocimiento de los hallazgos y por lo tanto, la aplicación de éstos a la práctica.

\section{La evaluación de las revistas.}

El concepto de las revistas núcleo surgió como resultado de los primeros estudios bibliométricos con Samuel Bradford en 1940. En los cuales observó que había un grupo de revistas que acumulaba más citas que los demás. A estas revistas se les denominó núcleo ${ }^{5}$. Esto significa que hay un grupo de revistas cuya producción es más visible y los autores que en ellas publican tienen más posibilidades de ser leídos y citados. De hecho, la revista y su calidad es lo que aumenta la visibilidad para los investigadores y autores.

\section{Los indicadores más utilizados en la evaluación de las revistas-}

Los indicadores más utilizados en la evaluación de las revistas científicas son: Bibliométricos, de productividad, de visibilidad, de estabilidad y obsolescencia, de calidad editorial, de calidad científica, como a continuación se presentan:

El tratamiento y manejo de la literatura científica por medios cuantitativos de recuento y análisis no sólo sirve para el volumen de publicaciones, la productividad de autores, o materias, sino también en un sentido más amplio, para el conocimiento de los procesos y la naturaleza de las ciencias.

- Indicadores de Productividad. Los indicadores de productividad hacen referencia al análisis de la revista por el número de revistas producidas por año, de artículos publicados por año, de artículos por revista, del tipo de artículos: enfermería clínica, docencia, administración e investigación. Además se contempla en la productividad el número de artículos de opinión, de artículos publicados por autor, en coautoría, por nivel académico del los autores, por lugar de procedencia: por país, por Estado, artículos revisados y rechazados por año. etc. 
- Indicadores de Visibilidad. Los indicadores de visibilidad hacen referencia al análisis de la revista de acuerdo con el uso, difusión, impacto y posicionamiento de la misma fuera de la institución donde se produce. Esto implica: el número de inscripciones de otros países, suscripciones por país, en Escuelas y Facultades de enfermería e instituciones hospitalarias, en otros ámbitos educativos y de salud, suscripciones de profesionales de enfermería, de otros profesionales ajenos a la enfermería y países donde se difunde la revista.

- Indicadores de estabilidad y obsolescencia. Los indicadores de estabilidad hacen referencia a la periodicidad de la publicación, entendida como el número de fascículos que se publican en un tiempo determinado y los indicadores de obsolescencia hacen referencia al análisis de la revista de acuerdo con la vigencia de los artículos e implica: Fechas de publicación, referencias del artículo, media de tiempo entre la recepción y la publicación del artículo, así como los años de publicación de las citas.

- Indicadores de calidad editorial. Los indicadores de calidad editorial hacen referencia al análisis de la revista que incluye el cumplimiento de los siguientes puntos: Número internacional normalizado para publicaciones seriadas (ISSN Internacional Standart Serial Number), sí el Comité Editorial está integrado por profesionales reconocidos en la disciplina que tienen la escolaridad necesaria, la filiación institucional y si son los necesarios para esta labor, que la dirección editorial sea diferente al Director de la institución en donde se edita la revista, que las características del proceso de arbitraje sean rigorosas, especializadas y está a cargo de árbitros nacionales e internacionales. Además la calidad editorial implica que los resultados de las evaluaciones se entreguen por escrito, se envíen los resultados del arbitraje por correo postal o electrónico, se especifique la cobertura temática y el público al que va dirigido, se identifique si hay mayor proporción entre los artículos por invitación, que se presente la tabla de contenidos en español e inglés, que se explicite la filiación institucional de las o los autores, si los autores pertenecen a diversas instituciones nacionales y extranjeras, que los ejemplares estén numerados, que en algún apartado se encuentren las instrucciones para las y los autores, y que exista el membrete bibliográfico en cada artículo y pagina. ${ }^{7}$

- Indicadores de calidad científica. Los indicadores de calidad científica hacen referencia al análisis de la revista de acuerdo a las aportaciones que hace al conocimiento y desarrollo de la disciplina. Para determinar la calidad científica se debe precisar si la revista sirve como vínculo de comunicación y discusión de resultados de investigación científicos y de desarrollo tecnológicos para la disciplina de enfermería, si los artículos son certificados (evaluados por pares), si los artículos son arbitrados (evaluados por consultores editoriales o por especialistas en la materia anónimos), si la revista incluye manuscritos: reportes de investigación científica, artículos de reflexión, documentos originales que presenten los resultados de estudios efectuados por el autor o autores respecto de un problema de índole teórico o de práctica diaria), si incluyen artículos de revisión (estudios que provienen del análisis profundo del estado del arte del tópico a tratar, si incluyen otro tipo de manuscritos como: notas cortas de un tema específico del dominio disciplinar, ponencias, trabajos presentados en congresos, coloquios, considerados por el editor/a como contribuciones originales a la disciplina, reseñas bibliográficas, artículos que presentan una revisión crítica de la literatura que es de interés para la disciplina) artículos editoriales que representan la posición del editor o del Comité Editorial y otros tópicos que no estén incluidos en estos apartados, como tesis de grado. ${ }^{10}$

- Indicadores de infraestructura. Esta dimensión se refiere a los recursos de que se dispone para operar la revista e implican: Número de recursos humanos, recursos físicos de los que se disponen, recursos materiales y financieros necesarios para la realización de la compleja actividad editorial.

\section{MATERIAL Y MÉTODOS}

La variables medidas fueron: Productividad, Visibilidad, Estabilidad y obsolescencia, Calidad Editorial, -Calidad Científica e Infraestructura. El tipo de investigación fue descriptiva, analítica, retrospectiva, longitudinal y de corte cuantitativo. El diseño fue no experimental. El estudio consistió en una evaluación interna y externa de la producción científica de la Revista Enfermería Universitaria y se llevó a cabo en tres etapas: En la primera, se estructuró el proyecto con los estados del arte de los estudios de evaluación científica de las revistas de enfermería nacionales e internacionales y se construyeron dos cuestionarios tipo Likert con los indicadores correspondientes y con base en la revisión de la literatura. En la segunda, se realizó la recolección, el procesamiento y el análisis de los datos para integrar la evaluación interna y externa de la revista y en la tercera y úl- 
tima etapa se integró el informe y el reporte de investigación para publicación.Las técnicas e instrumentos de recolección fueron: fichas de trabajo, entrevista y cuestionarios y una guía de observación de indicadores. La evaluación interna se realizó a través de la aplicación de una guía con 9 indicadores que evalúo las dimensiones de bibliometría, visibilidad, estabilidad, obsolescencia, de calidad editorial y científica de nueve revistas publicadas en los años 2004, 2005 y 2006. La evaluación externa se efectuó con dos cuestionarios que sirvieron de base, uno para las y los lectores y otro para las y los autores que han escrito en la revista con el fin de conocer su opinión. Se estructuró cada cuestionario con 47 preguntas de las cuales 46 fueron cerradas y una abierta. De estas 7 preguntas fueron para los datos sociodemográficos, 3 para los datos de identificación de las revista, 12 para la calidad científica, 13 para la calidad editorial, 11 para la calidad del diseño editorial y una abierta para recabar las sugerencias y comentarios. El universo Población y muestra: Para la evaluación interna el universo lo constituyeron 9 ejemplares de la revista Enfermería Universitaria editadas en los años 2004, 2005 y 2006 . Este universo fue el 100\% de la muestra. Para la evaluación externa se enviaron por vía postal 400 cuestionarios a las lectoras/es y aplicaron 80 a las y los autores. Se excluyeron las revistas publicadas posteriores al año 2006, los lectores y autores que no quisieron participar en la investigación y los cuestionarios incompletos o los que no se enviaron. La muestra fue de 50 autores 39, del D.F. 10 a nivel nacional y 1 internacional. De igual forma se entrevistaron a 116 lectoras y lectoras del Distrito Federal de diversos estados de la República Mexicana y de nivel internacional.

Las consideraciones éticas: Para este estudio se consideró el principio del anonimato y privacidad del reglamento de la Ley General de Salud en materia de investigación para la salud, titulo $2^{\circ}$, cap. I, Art. 10, Fracc. V que se refiere al consentimiento informado de los sujetos implicados en una investigación y el principio de privacidad del Art. 16 que hace referencia al anonimato de los sujetos de investigación, por lo que no se solicitó el nombre de las y los lectores y de las y los autores y se les respetó su libertad de decidir participar o no en la investigación.

\section{RESULTADOS:}

\section{Evaluación interna de las dimensiones.}

- Productividad. Se publicaron 9 ejemplares de la revista, 80 artículos, de los cuales 210 son de enfermería y 403 de otras áreas o disciplinas de la salud 26 artículos por año de 193 autoras/es conformados por profesorado y alumnado de la ENEO-UNAM principalmente y en una mínima proporción por autoras/es de otras Universidades e Instituciones de salud del país y del extranjero ( ver cuadro 1). En estos años la revista incluyó manuscritos de editoriales, investigaciones científicas y artículos de reflexión originales, reportes de tesis, notas, etc, distribuidos en 7 secciones: La Editorial, reporte de investigación, práctica profesional, trabajo colegiado, los maestros escriben, los alumnos escriben, algo para reflexionar y un apartado de instrucción para autoras/es. Así mismo se publicaron 12 artículos de docencia, 12 de aspectos de salud, 3 de salud reproductiva y 12 de investigación. 613 referencias citadas, el número promedio por artículo por ejemplar fue de 7 con un intervalo de 3.9 a 10.4. El número total de referencias citadas reportadas en los 9 números fue de 613, con un mínimo de 31 en el primer número de la revista y un máximo de 104 en el número 9, reflejando un ascenso paulatino de citas por año. (Ver cuadro 2) El número de referencias de otros profesionales diferentes de enfermería fue mayor que el de citas de enfermería con un total de 403 y 210 respectivamente. El número de citas fue de 29 , el idioma en que se cita fue predominantemente el español con un total de 529 seguido por el inglés con 83 . Hubo solo una cita en portugués y el estilo de elaboración de citas es Vancouver, el promedio de palabras por título por ejemplar fue de 12 con un intervalo que va de 9 a 15.6, el número de títulos de enfermería fue de 44 y alusivo a otras disciplinas 36.

- Visibilidad. La revista fue el principal recurso de difusión científica del profesorado y alumnado de la ENEOUNAM. A su corta edad fue vínculo de comunicación del

\section{Cuadro $\mathrm{N}^{\circ} 1$ \\ Número de artículos publicados por año en la Revista Enfermería Universitaria editados durante los años 2004-2006 En méxico, D. F.}

\begin{tabular}{|lc|c|c|}
\hline \multirow{2}{*}{ O P C I O N E S } & \multicolumn{3}{|c|}{$\begin{array}{c}\text { No. de artículos } \\
\text { publicados por año }\end{array}$} \\
\cline { 2 - 5 } Año de edición & \multicolumn{2}{|c|}{ F } & $\%$ \\
\hline 1. & 2004. & 26 & 32.50 \\
2. & 2005. & 25 & 31.25 \\
\multirow{2}{*}{3.} & 2006. & 27 & 33.75 \\
\hline \multicolumn{2}{|l|}{ T O T A L E S } & 80 & $100 \%$ \\
\hline
\end{tabular}

Fuente: Observación directa de la revisión de los 9 ejemplares de la Revista Enfermería Universitaria publicados del 2004 al 2006 durante agosto-marzo del 2006-2007. 


\section{Cuadro $\mathrm{N}^{\circ} 2$ \\ Número de citas por revista de los ejemplares \\ de la revista enfermería universitaria editados durante los años 2004-2006 \\ En México, D. F.}

\begin{tabular}{|l|l|l|l|}
\hline \multicolumn{3}{|c|}{ REVISTA } & \multicolumn{1}{|c|}{$\begin{array}{l}\text { No de } \\
\text { citas }\end{array}$} \\
\hline 1 & $\begin{array}{l}\text { AÑO 1, Vol 1, No.1 Enero-Marzo, } \\
2004 \text { 32 pp. }\end{array}$ & 31 & 5,06 \\
\hline 2 & $\begin{array}{l}\text { AÑO 1, Vol 1 No.2 Mayo-agosto } \\
2004 \text { 44 pp. }\end{array}$ & 55 & 8,97 \\
\hline 3 & $\begin{array}{l}\text { AÑO 1, Vol 1 No.3 Sep-Dic 2004 } \\
44, \text { pp. }\end{array}$ & 58 & 9,46 \\
\hline 4 & $\begin{array}{l}\text { AÑO 2, Vol 2, No.1 Enero-Marzo } \\
2005 \text { 48 pp. }\end{array}$ & 83 & 13,54 \\
\hline 5 & $\begin{array}{l}\text { AÑO 2, Vol 2 No.2 Mayo-agosto } \\
2005 \text { 40 pp. }\end{array}$ & 48 & 7,83 \\
\hline 6 & $\begin{array}{l}\text { AÑO 2, Vol 2 No.3 Sep-Dic 2005 } \\
55 \text { pp. }\end{array}$ & 84 & 13,7 \\
\hline 7 & $\begin{array}{l}\text { AÑO 3, Vol 3, No.1 Enero-Abril } \\
2006 \text { 48 pp. }\end{array}$ & 68 & 11,09 \\
\hline 8 & $\begin{array}{l}\text { AÑO 3, Vol 3, No.2 Mayo-Agosto } \\
2006 \text { 56 pp. }\end{array}$ & 82 & 13,38 \\
\hline 9 & $\begin{array}{l}\text { AÑO 3, Vol 3, No 3 Sep-Dic 2006 } \\
56 \text { pp. }\end{array}$ & 104 & 16,97 \\
\hline & \begin{tabular}{l} 
Total \\
\hline
\end{tabular} & 613 & 100 \\
\hline
\end{tabular}

Fuente: Misma del cuadro No. 1

pensamiento de enfermero en el país, principalmente dentro del Distrito Federal y de algunos Municipios del Estado de México. Su cobertura incluyó a otros países como Estados Unidos, España y Colombia, con un tiraje de mil ejemplares.

- Dimensión de estabilidad y obsolescencia. La revista fue publicada de manera ininterrumpida cada 4 meses a partir del 2004, produciéndose tres ejemplares por año. Los artículos fueron vigentes de acuerdo a los años de las citas referidas.

- Calidad científica. Se evaluó que la revista "Enfermería Universitaria" se ha ido constituyendo un recurso valioso para el desarrollo del conocimiento de la disciplina de Enfermería en México. Es necesario destacar en la evaluación que la mayoría de los artículos no fueron resultado de investigaciones originales aunque si se incluyeron en la revistas artículos de interés general con calidad. Los datos más relevantes de la calidad científica arrojaron que el $85 \%$ consideró que la revista es un medio de comunicación entre las enfermeras y enfermeros, el 75\% opinó que la información que tiene la revista es relevante, el 75\% que los artículos incluyen las teorías de enfermería, el 70\% que si existen marcos conceptuales y estados del arte que fundamentan la disciplina de enfermería, el 85\% que si hay artículos de revisión de calidad, el 100\% que si hay artículos de reflexión, el $75 \%$ que la revista no incluye artículos de investigación de tesis de grado de enfermería y el $65 \%$ que sólo algunas veces la revista presenta aportaciones al desarrollo disciplinar.

- Dimensión de calidad editorial. La revista tuvo el Número Internacional Normalizado para Publicaciones Seriadas (ISSN-International Standar Serial Number) No. 1665-7063. La revista fue indexada en la base de datos CUIDEN de Granada España, en el Sistema de Información LATINDEX del Departamento de Bibliografía Latinoamericana de la Dirección General de Bibliotecas de la UNAM y está en proceso de indexarse en las bases de SCIELO, ARTEMISA y LILACS. Está en proceso de digitalizarse una vez que se hagan las mejoras editoriales. Se integró un Comité Editorial conformado principalmente por profesorado de la ENEO con reconocimiento académico y con grado académico de Licenciatura, Maestría ó Doctorado. La Dirección Editorial y la dirección de la institución la ocuparon personas distintas.

- Dimensión de estructura: Durante los años 2004-2006 se contó con un cubículo pequeño, 1 archivero, 2 gavetas, 1 escritorio, 3 sillas secretariales, 1 computadora con Internet y teléfono. En cuanto a los recursos humanos se tuvieron los requerimientos mínimos indispensables para la producción editorial, y en cuanto a los recursos humanos que han participado en la revisión y edición de la revista del 2004 al 2006, fueron 45 personas: 3 directivos, 4 consejeras editoriales, 18 integrantes del Comité editorial, 7 integrantes del Comité Asesor y 2 integrantes de la Cátedra "Javier Barros Sierra". En cuanto a los aspectos financieros, durante los años 2004-2006 se produjeron 9000 ejemplares de 9 números diferentes de la revista Enfermería Universitaria, el costo total de producción fue de $\$ 338,300.00$ pesos y el costo total de envío postal fue de $\$ 47,735.17$, sumando un total de $\$ 386,565.17$, con un costo unitario promedio por revista de $\$ 42.95$.

\section{Evaluación externa desde la perspectiva de las y los autores.}

De 77 artículos publicados en los 9 ejemplares la revista Enfermería universitaria durante los años 2004, 2005, 2006, se presentan resultados con una muestra de 50 autoras y 
autores de un total de 183 de la primera a la sexta autoría, incluyendo: 80 autores principales, 40, $2^{\text {dos }}$ autores, $283^{\text {eros. }}$ autores y 35 autores de la $4^{\mathrm{a}}$ a la $6^{\mathrm{a}}$ autoría, encontrándose los siguiente:

Referente a los datos generales de las y los autores. El 98\% de las y los entrevistados fueron enfermeras y sólo el $2 \%$ son hombres con profesiones del área de la salud. (ver cuadro 3) Referente a la edad, el $85 \%$ tuvo entre 41 a 60 años, un $60 \%$ con grado de maestría, el $10 \%$ estudios de doctorado y $15 \%$ licenciatura, el $90 \%$ de su actividad principal la realizan en el área de docencia e investigación. El $70 \%$ de está inscrito en la revista desde el 2004 y le llega de manera gratuita, el 65 \% tiene filiación institucional con la ENEO-UNAM, un $30 \%$ proceden de diversos estados de la república y un $5 \%$ de procedencia internacional.

Relativo al año, volumen y número de la revista a evaluar. Respecto al año de la revista evaluada por los lectores, el $50 \%$ fue del 2004 , el $30 \%$ del 2005 y el $10 \%$ del 2006 . Del volumen del ejemplar evaluado el 60\% fue del número 3, el $26 \%$ del 2, y el $14 \%$ del 1. En relación al número del ejemplar evaluado el $66 \%$ fue del número 3 , el $20 \%$ del 1 y el $14 \%$ del 2 .

Respecto a la dimensión de calidad científica de la Revista Enfermería Universitaria opinan lo siguiente: El $70 \%$ consideró que es un medio de comunicación entre las Enfermeras/os y sólo el $5 \%$ consideró que parcialmente le aporta algunos elementos a la enfermería, el 68 \% refirió que mayoría de la información es relevante y un $8 \%$ que parcialmente aporta algunos elementos a la Enfermería. El $68 \%$ indicó que la mayoría de la información que difunde es original y un $8 \%$ que pocos artículos son originales. El 70\% consideró que sólo algunos artículos incluyen las teo-

\section{Cuadro No. 3}

Distribución de la profesión de las autoras y autores que escriben en la Revista Enfermería Universitaria durante los años 2004, 2005 y 2006 en México, D. F.

\begin{tabular}{|l|l|l|}
\hline \multicolumn{1}{|c|}{ Profesión } & \multicolumn{2}{c|}{ TOTAL } \\
\hline \multicolumn{1}{|c|}{ O P C I O N E S } & \multicolumn{1}{c|}{ F } & \multicolumn{1}{c|}{$\%$} \\
\hline $\begin{array}{l}\text { 1. Enfermera/o. } \\
\text { 2. Médica/o. }\end{array}$ & 46 & 92,00 \\
$\begin{array}{l}\text { 3. Otro profesional del área } \\
\text { de ciencias de la salud } \\
\begin{array}{l}\text { 4. Otro profesional diferente } \\
\text { del área de ciencias } \\
\text { de la salud }\end{array} \\
\text { 5. Sin respuesta. }\end{array}$ & 1 & 2,00 \\
\hline T O T A L E S & 0 & 6,00 \\
\hline
\end{tabular}

Fuente: Misma del cuadro No. 1 rías de Enfermería y un 30 \% consideró que parcialmente algunos si las incluyen. El $66 \%$ consideró que solo algunos artículos incluyen marcos conceptuales y estados del arte que fundamente la disciplina de enfermería y un $34 \%$ que no los incluye. El 90\% refirió que se difunden reportes de investigación científica de calidad. El 66\% manifestó que la revista solo algunas veces incluye artículos de revisión de calidad de tópicos de Enfermería y el 34\% refirió que siempre o casi siempre se incluyen estos artículos. El 100\% dijo que la revista siempre o casi siempre incluye artículos de reflexión de calidad. El 100\% indicó que la revista siempre o casi siempre incluye artículos editoriales de interés para la Enfermería. El 70 \% señaló que la revista no incluye artículos de investigación de tesis de grado de Enfermería. El 66 \% consideró que sólo algunas veces la revista presenta aportaciones al desarrollo disciplinar en tanto que un 34\% refirió que siempre o casi siempre lo hace. Respecto a la pregunta de si la revista le aporta a otras disciplinas, el 55\% no respondió el 25 \% consideró que los artículos son de interés para otras disciplinas además de Enfermería y el 20\% dijo que no aporta a otras disciplinas.

Respecto a la dimensión de calidad editorial de la Revista Enfermería Universitaria opinan lo siguiente: El 88 \% consideró que la mayoría o todos los artículos son evaluados objetivamente por pares, en tanto que el 12\% indicó que sólo algunos son evaluados objetivamente por pares. El 76 \% consideró que la mayoría o todos los artículos son arbitrados por consultores editoriales o especialistas de la disciplina en tanto que el $24 \%$ consideró que sólo algunos no son evaluados por consultores editoriales o especialistas. Respecto al anonimato del arbitraje, el 50\% no respondió y el otro 50 $\%$ dijeron que en su mayoría fueron arbitrados de manera anónima. En relación con la procedencia de los consultores editoriales que realizan el arbitraje de sus artículos consideró que el $80 \%$ son de procedencia local, nacional e internacional. Respecto a la entrega de resultados del arbitraje, el 56 $\%$ no respondió, el $40 \%$ consideró que se hace por escrito de forma personalizada y un 4 \% indicó que es verbal. En relación con la oportunidad de entrega de los resultados de arbitraje el $90 \%$ indicó que si es oportuna, pues en una plazo máximo de dos meses se tienen los resultados del arbitraje. Respecto a la pregunta de que sí los artículos que escriben en la revista han sido por invitación o por iniciativa propia, el 50 \% no respondió y el otro 50 \% indicó que la mayoría ha sido por iniciativa propia y sólo algunos por invitación. Respecto a las secciones temáticas que incluye la revista el 90\% consideró que son suficientes o las apropiadas para el tipo 
de artículos que publica, un $4 \%$ indicó que se deben incluir otras secciones para tesis o artículos de revisión y un $6 \%$ consideró que no son las más apropiadas. El lugar de procedencia de las/os autores fue el 20\% nacional, 2\% internacional y local 78\% (D.F 18\% y ENEO-UNAM 60\%). En relación con los criterios para la publicación de artículos en la revista, el $66 \%$ consideró que son parcialmente los óptimos, el 30 $\%$ que son óptimos y un $4 \%$ no respondió. Referente a la claridad de los criterios, el 92\% refirió que son muy claros.( ver cuadro 4) El 96\% manifestó que le gustaría que la revista estuviera disponible en Internet, pues mejoraría la difusión de artículos. Respecto a la pregunta de que si le gustaría seguir publicando en la revista, el 90 \% indicó que si y sólo el $10 \%$ indicó que ocasionalmente.

Respecto a la dimensión de diseño editorial de la Revista Enfermería Universitaria opinan lo siguiente: Respecto al diseño editorial, el 50 \% indicó que el diseño informativo y gráfico es apropiado y el otro 50\% manifestó que es parcialmente adecuado y requiere mejoras, En relación con el color en escala de grises el $100 \%$ consideró que es el adecuado para el tipo de publicación. Con respecto al tipo de letra y tamaño, 100 \% consideró que la letra Arial 12 es la más apropiado para favorecer la lectura. Respecto a la suficiencia de los espacios asignados a cada sección, el 50 \% no respondió, el $42 \%$ indicó que son suficientes y responden a sus intereses. Referente a si las secciones responden a sus intereses de difusión, el 96\% indicó que son suficientes. Respecto al diseño de la portada, el 55 \% opinó que en ocasiones es atractiva y acorde a la temática, el $45 \%$ indicó que es atractiva y alude a las temáticas de los artículos. En relación con las imágenes incluidas en la revista, el $55 \%$ refirió que a veces tienen relación con los artículos y el $45 \%$ restante opinó que siempre y casi siempre tienen relación. En relación con los cuadros y figuras, el $100 \%$ indicó que apoyan el contenido de los artículos y se entienden sin hacer referencia al texto. Al $95 \%$ le llegó la revista de manera periódica y oportuna y sólo el 5 $\%$ no la recibió con oportunidad. Respecto a si han recibido ejemplares de obsequio por ser autoras/es, el $45 \%$ indicó que sí y el $55 \%$ que no.

\section{Evaluación externa desde la perspectiva de las y los lectores.}

De 116 lectores entrevistados de un total de 400 cuestionarios enviados se obtuvieron los siguientes resultados:

Referente a los datos generales de las y los lectores. El 90.52\% fueron Enfermeras/os, 9.48\% otros profesionales de la salud. El $94.83 \%$ tiene entre 41 y 60 años. El $81.9 \%$ fueron mujeres y el $18.1 \%$ hombres. El $72.41 \%$ tiene el grado de maestría, el $9.48 \%$ estudios de doctorado y $18.1 \%$ licenciatura. El 69.82 $\%$ de su actividad principal la realizan en el área de docencia e investigación. El 64.6\% está inscrito en la revista de los cuales el $21.5 \%$ paga cuota anual y al $43.1 \%$ le llega de manera gratuita. El $44.83 \%$ lleva 3 años de estar suscrito, el $10.3 \%$ menos de un año y del $44.8 \%$ restante no se obtuvo respuesta.

Relativo al año, volumen y número de la revista a evaluar. Respecto al año de la revista evaluada por los lectores, el $18.1 \%$ fue del 2004 , el $8.6 \%$ del 2005 , el $54.6 \%$ del 2006 y el restante $18.9 \%$ no dio respuesta. $65 \%$ tiene filiación insti-

\section{Cuadro $\mathrm{N}^{\circ} 4$}

Distribución de la opinión de las autoras y autores que escribieron en la Revista Enfermería Universitaria durante los años 2004, 2005 y 2006 respecto de la claridad de los criterios de publicación de la revista

\begin{tabular}{|c|c|c|}
\hline $\begin{array}{c}\text { Opinión respecto a la claridad de los criterios } \\
\text { para la publicación de artículos }\end{array}$ & \multicolumn{2}{|c|}{ TOTAL } \\
\hline OPCIONES & $\mathbf{F}$ & $\%$ \\
\hline $\begin{array}{l}\text { 1. Me parece que explican con muchas claridad y detalle la información } \\
\text { que debe ir en cada parte del artículo. }\end{array}$ & 8 & 16,00 \\
\hline $\begin{array}{l}\text { 2. Me parece que la mayoría de los criterios son muy claros, pero otros no, } \\
\text { se tendría que precisar más la información que se solicita }\end{array}$ & 38 & 76,00 \\
\hline $\begin{array}{l}\text { 3. Me parece que parcialmente los criterios tienen claridad, } \\
\text { pues me generan algunas dudas. }\end{array}$ & 2 & 4,00 \\
\hline 4. Me parece que no son claros los criterios para la aceptación de artículos. & 2 & 4,00 \\
\hline 5. Sin respuesta. & 0 & - \\
\hline TOTALES & 50 & $100 \%$ \\
\hline
\end{tabular}

Fuente: Misma del cuadro No. 1 
tucional con la ENEO-UNAM, un 30\% proceden de diversos estados de la república y un $5 \%$ de procedencia internacional. Del volumen del ejemplar evaluado el $53.45 \%$ fue del número 2 , el $28.45 \%$ del 3 y el $18.1 \%$ del 1 . En relación al número del ejemplar evaluado el 35.3\% fue del número 3 , el $27.5 \%$ del 1 , el $8.6 \%$ del 2 y el $28.4 \%$ no dio respuesta.

Respecto a la dimensión de calidad científica de la Revista Enfermería Universitaria opinan lo siguiente: El 81.9\% consideró que es un medio de comunicación entre las Enfermeras/os. El 90.52\% indicó que le aportó información relevante, actualizada y científica. El 81.9\% señaló que la mayoría de la información que difunde es original y el $18 \%$ que pocos artículos son originales. El 53.45\% refirió que parcialmente los artículos incluyen las teorías de Enfermería y el $46.5 \%$ comentó que la mayoría si las incluyen. El 62.07\% consideró que parcialmente algunos artículos incluyen marcos conceptuales y estados del arte y el $37.93 \%$ si los incluyen. El 81.9\% manifestó que siempre o casi siempre se incluyen artículos de investigación científica de calidad. El 81.9\% consideró que siempre o casi siempre incluyen artículos de calidad. El $72.4 \%$ dice que la revista siempre o casi siempre incluye artículos de reflexión de calidad de tópicos de Enfermería. El $73.2 \%$ dice que la revista siempre o casi siempre incluye artículos editoriales de interés para la Enfermería. El $54.3 \%$ señala que la revista no incluye artículos de investigación de tesis de grado de Enfermería. El 72.4\% consideró que siempre o casi siempre la revista aporta elementos al desarrollo disciplinar. El 72.4\% consideró que la mayoría de los artículos aportan información a otras disciplinas.

Respecto a la dimensión de calidad editorial de la Revista Enfermería Universitaria opinan lo siguiente: El 72.4\% considera que la mayoría o todos los artículos son evaluados objetivamente por pares, en tanto el $27.5 \%$ indican que sólo algunos son evaluados. El $72.4 \%$ considera que la mayoría o todos los artículos son arbitrados por consultores editoriales o especialistas de la disciplina en tanto que el $26.7 \%$ consideran que sólo algunos son evaluados. El 90.5\% de los lectores refiere que los artículos son arbitrados de manera anónima. En relación con la procedencia de los consultores editoriales que realizan el arbitraje el $45.6 \%$ considera que es local, nacional e internacional. Respecto a la entrega de resultados del arbitraje, el 36.2\% considera que se hace por escrito de forma personalizada y el 36.2\% indicó que se realiza por correo electrónico. En relación con la oportunidad de entrega de los resultados de arbitraje el $55.1 \%$ indica que si es oportuna en un plazo máximo de 30 días y el 35\% refiere que es en un plazo máximo de 2 meses. Respecto a la pregunta de que sí los artículos que escriben en la revista han sido por invitación o por iniciativa propia, el otro $25 \%$ indicó que fue por iniciativa propia. Respecto a las secciones temáticas que incluye la revista el $81.8 \%$ consideró que son suficientes o las más apropiadas para el tipo de artículos que publica. Según la procedencia de los lectores que han escrito en la revista, el $28.4 \%$ son nacionales, el $1 \%$ internacional y $70.6 \%$ local. En relación con los criterios para la publicación de artículos en la revista, el 37\% consideran que son óptimos y el $44.8 \%$ que son parcialmente óptimos. El 81\% manifiesta que le gustaría que la revista estuviera disponible en Internet para mejora de la difusión de los artículos. Respecto a la pregunta de que si le gustaría seguir publicando en la revista, el 76.74\% respondió afirmativamente.

Respecto a la dimensión de diseño editorial de la Revista Enfermería Universitaria opinan lo siguiente: Respecto al diseño editorial, el 99.1\% indicó que el diseño informativo y gráfico es el más apropiado para la publicación. En relación con el color en escala de grises el 100\% consideró que es el más adecuado para el tipo de publicación. Con respecto al tipo de letra y tamaño, 100\% consideró la letra Arial 12 como la más apropiada para favorecer la lectura. Respecto a la suficiencia de los espacios asignados a cada sección, el 100\% indicó que son suficientes. Respecto al diseño de la portada, el $100 \%$ opinó que es atractiva. En relación con las imágenes incluidas en la revista, el 100\% refieren que éstas son alusivas a los artículos publicados. En relación con los cuadros y figuras, el $100 \%$ indica que apoyan el contenido de los artículos y se entienden sin hacer referencia al texto. Al 95\% le llega la revista siempre de manera periódica y oportuna y sólo el $5 \%$ la ha recibido con cierta oportunidad. Respecto a si han recibido ejemplares de obsequio por ser autoras/es, el $45 \%$ indicó que sí y el 55\% que no.

La revista Enfermería Universitaria ha sido desde su creación en el 2004, un recurso valioso para el desarrollo del conocimiento de la enfermería en México. La aportación de la ENEO con la revista a la comunidad científica de la Enfermería ha sido vehículo para la difusión del conocimiento de la enfermería nacional e internacional. Este esfuerzo editorial ha sido valioso no sólo porque permite difundir el conocimiento del profesorado y alumnado y de las enfermeras y enfermeros de México, sino porque la ENEO ha mantenido la revista vigente, la ha mejorado y la ha fortalecido, haciendo de ella una revista de consulta en el campo de la enfermería en español, con visibilidad en México y en algunos países del extranjero y con calidad editorial. 


\section{CONCLUSIONES}

La evaluación realizada de la revista arrojó datos muy positivos en todas las dimensiones e indicadores medidos de la evaluación interna y externa realizada. Sin embargo, la única evaluación que requiere fortalecerse y mejorarse es la de la calidad científica y de infraestructura. Para ello es necesario fortalecer la revista con artículos producto de investigaciones de enfermería, incrementar el grupo editorial que coadyuve en la gestión de la revista con recursos humanos capacitados, dar cabida a autores de otras revistas para fortalecer los artículos de investigación en el campo de la docencia, la administración y los servicios de instituciones nacionales y del extranjero. Es necesario además contar con un equipo experimentado de profesionales para evaluación de pares, lograr la indexación de la revista y también su auto-financiamiento. Finalmente será necesario para el 2010, realizar otra evaluación que permita contar con datos reales del avance y de los logros de la revista y de esa manera asegurar su calidad editorial y científica.

\section{REFERENCIAS BIBLIOGRÁFICAS:}

1 Amezcua, Manuel y Cols. La pequeña ciencia: producción, repercusión y transferencia del conocimiento. La enfermería del área lingüística del español y del portugués. Documento impreso. Fundación Index. Granada, 2006. p. 7.

2 Amezcua, Manuel y Cols. Ibid. p 9.

3 Amezcua, Manuel y Cols. Ibid. p. 8

4 Benavides-T; Raquel. La investigación en la enfermería Mexicana: Análisis del contexto nacional e internacional. En: Revista Enfermería del IMSS, No. 10 (3), Sep-Dic. México, 2002. p. 153.

5 Grupo de Estudios Documentales. Las Revistas núcleo de enfermería: La elección de la mejor revista para publicar un trabajo. Fundación Index. Documento impreso. Granada, 2007. p. 7

6 Barranco-Armeo y Cols. Análisis cienciométrico de la revista Enfermería Global 2002-2004. Documento impreso. Madrid, 2007, p.2

7 Jiménez-S, Juana. "Algunas consideraciones sobre la evaluación de la calidad de las revistas"; en: Revista de Enfermería de IMSS, No. 1 Vol. 11, enero-abril, México, 2003. pp. 1-3.

8 lbid. p. 1-2.

\section{OTRAS REFERENCIAS DE CONSULTA:}

Blanco Pérez, Azucena González Guitián, Carlos y Touriño Míguez, Elvira. El grado de cumplimentación de las normas editoriales internacionales por parte de las revistas de enfermería. Enfermería Clínica. 1994 jul-ago. 4(4):162-165. Original, artículo.

ENEO. Enfermería Universitaria . Revista No. 1 Vol. 1 Año 1 , ene-marzo, México, 2004, 32 pp.

ENEO. Enfermería Universitaria. Revista No. 2 Vol. 1 Año 1, mayo-agosto, México, 2004, 44 pp.

ENEO. Enfermería Universitaria. Revista No. 3 Vol. 1 Año 1, dicenero, México, 2004, 44 pp.

ENEO. Enfermería Universitaria. Revista No. 1 Vol. 2 Año 2, enemarzo, México, 2005, 48 pp.

ENEO. Enfermería Universitaria. Revista No. 2 Vol. 2 Año 2, mayo-agosto, México, 2005, 40 pp.

ENEO. Enfermería Universitaria. Revista No. 3 Vol. 2 Año 2, dicenero, México, 2005, 55 pp.

ENEO. Enfermería Universitaria. Revista No. 1 Vol. 3 Año 3, enemarzo, México, 2006, 48 pp.

ENEO. Enfermería Universitaria. Revista No. 2 Vol. 3 Año 3, mayo-agosto, México, 2006, 56 pp.

ENEO. Enfermería Universitaria. Revista No. 3 Vol. 3 Año 3, dicenero, México, 2006, 56 pp.

Ferreiro, L. Bibliometría, análisis biobliométrico. Ed. Eypasa, Madrid, 1993.

Gálvez Toro, Alberto Cols. Impacto 2002 de las revistas españolas de enfermería y su evolución en los últimos diez años. Index Enferm. Granada, España. 2003. Otoño XII(42):73-77.

Gálvez Toro, Alberto Hueso Montoro, César y Amezcua, Manuel. Consumo de información de las Revistas de Enfermería del área lingüística del español y portugués (año 2002) Desarrollo Científico de Enfermería. México 2004. Abril. 12(3):69-76. Gálvez Toro, Alberto y Cols. Indicadores CUIDEN de repercusión de las revistas de enfermería. Index Enferm. Granada, 2004. Otoño XIII(46):76-80.

Gálvez Toro, Alberto y Cols. Envejecimiento de las revistas de enfermería del área lingüística del español y del portugués. Evidentia. Madrid, 2005. sep-dic. 2(6).

Gálvez Toro, Alberto Cols. Evaluación de la actividad científica de la enfermería española. Impacto y aislamiento en el año 2000. Index Enferm. Granada, 2001. Otoño XII(34):54-64.

Hidalgo-N; Adalia y cols. Análisis de las publicaciones de investigación de enfermería en México (1992-1995). $2^{2}$ parte. Desarrollo Científ Enferm. México, 1996. Feb. 4(2):16-23. Palazuelos-L; Caludine y Cols. Análisis de las publicaciones de investigación de enfermería en México (1987-1991). Desarrollo Científ Enferm. México, 1994. Dic. 2(12):13-20.

\section{DIRECCIÓN PARA CORRESPONDENCIA:}

Dra. Lasty Balseiro Almario:lastybals@hotmail.com 